\title{
Two-phase slug flows in helical pipes: Slug frequency alterations and helicity fluctuations
}

\author{
M. Gourma ${ }^{a, *}$, P. G. Verdin ${ }^{b}$ \\ ${ }^{a}$ Cranfield University, Energy 83 Power Engineering Institute. Cranfield MK43 OAL UK \\ ${ }^{b}$ Cranfield University, Oil \& Gas Engineering Centre. Cranfield MK43 OAL UK
}

\begin{abstract}
Air-water numerical simulations in the slug flow regime have been performed in horizontal helical pipes and the effects of geometries on the flow regime have been investigated. Depending on the length of the helix, outlet slug frequencies have been reduced with various degrees of efficiency. Correlations between mean tangential velocity and helicity density fluctuations have been identified and investigated qualitatively. These flow fields show smaller time scales than those obtained in volume fractions fluctuations. Shifts observed in the tangential velocity and mean helicity fluctuations to smaller time scales (high frequencies) are associated with regime transitions. For a slug flow undergoing a continuous transition to the annular flow regime, it is shown that the presence of slower (low frequencies) helicity fluctuations is attributed to the variations in the axial velocity. Finally, the analysis of the helicity at gas-liquid interface confirms the presence of the mixing zone at the slug front.
\end{abstract}

Keywords: Helical pipes, Slug flow, Slug frequency, Volume of fluid VOF, Helicity density, Helicity fluctuations

\footnotetext{
*Corresponding author.Tel:+44(0) 1234 755035, Fax:+44(0) 1234754746

Email address: m.gourma@cranfield.ac.uk (M. Gourma)
} 


\section{Introduction}

Helical coiled pipes are commonly used in industrial applications such as power generation, process plants, refrigeration and heat recovery systems, pharmaceutical and food industries. Related published work is available, such as the one from Naphon and Wongwises (2006), where the authors reviewed investigations on various flows in curved lines.

Pressure drop and two phase friction factors in helical lines has received considerable attention to predict transition to turbulent regimes, see Xin et al. (1997) and Zhao et al. (2003). Simulations from Vashisth and Nigam (2009) and Saffari and Moosavi (2014) predicted void fraction distribution, friction factors and velocity profiles as well as shear stress in vertical coil air-water flow systems. Murai et al. (2006) conducted experiments with a vertical set up; they concluded that the transition from bubbly to plug flow is quickened and that pressure fluctuations in the slugs body present lower amplitudes with higher frequencies than in gas bubbles. Experiments on boiling flow in small diameter coils by Cioncolini et al. (2008) showed that heating effects are adequately fitted using a modified Lockhart-Martinelli correlation. A similar approach was conducted by Mandal and Das (2002) with various coil diameters and flow rates when considering isothermal conditions.

To understand the physical mechanisms involved in the inception of turbulence in coiled devices, investigations have been carried out by Germano (1982). He concluded that at low Reynolds number, curvature and torsion appear to have first and second order effects on the transition to turbulent flows. Recently, Ciofalo et al. (2014) showed that torsion has little influence 
on turbulence, while an increase in curvature leads to confined fluctuations near outer wall regions.

It is acknowledged by Narasimha and Sreenivasan (1979), and Sreenivasan and Strykowski (1983), that transitions to turbulent flow regimes are delayed in helical lines and occur at Reynolds numbers well above the level required for a similar straight pipe. This is largely attributed to secondary re-circulations. Furthermore, numerical studies by Hüttl and Friedrich (2000) showed that turbulence is inhibited by high curvature. The work from Di Piazza and Ciofalo (2011) confirmed the occurrence of a transitional unsteady regime between the stationary and the turbulent state; they associated this intermediate state to Dean travelling waves.

The classical theories of turbulence are dominated by the concept of the energy cascade to the small scales as postulated by Kolmogorov (1941). However, it was discovered by Moreau (1961) and Moffatt (1969) that helicity has also an important role in the dynamics of fluids with large structures. It was shown that the joint cascade of both energy and helicity to small scales takes place if the system has large helical scales, see for example Brissaud et al. (1973) and Kraichnan (1973). Several scientists such as Rogers and Moin (1987), Polifke and Schtilman (1989), Levich et al. (1991), Yokoi and Yoshizawa (1993), Levich (2009), speculated that helicity fluctuations are fundamental to turbulence.

The control of flow regimes in pipelines is highly important for petrochemical industries. To that end, laboratory and field experiments are commonly carried out with various devices and tools to obtain accurate predictions. Slug flow regimes are frequently encountered at industrial scales. In the field, they are mainly due to the topology of the terrain (severe slugging) 
and may occur at low flow rates, (De Henau and Raithby, 1995). Slug flows are a concern for the integrity of the production facilities. Fabre and Liné (1992) explained that "the slug flow pattern results in sequences of long bubbles almost filling the pipe cross-section, successively followed by liquid slugs that may contain small bubbles". Studies in this field of research include the mathematical modeling of slug predictions, the experimental slug characterizations and the detailed mechanisms of slug inceptions. A comprehensive investigations on gas-liquid slug flows can be found in Dukler and Hubbard (1975), Moalem et al. (1991), Andreussi et al. (1993) and Kadri et al. (2010). An extended analysis is available in Hurlburt and Hanratty (1975). The intermittency and the statistical character of slug flow regimes are demonstrated and commonly recognised through various publications, see Nydal et al. (1992), Paglianti et al. (1996), Gopal and Jepson (1997) and van Hout et al. (2001).

Multiphase flow modelling and slug flow regimes (in particular in one dimensional codes) are widely approached using multi-fluid models, see Bonizzi and Issa (2003), Issa and Kempf (2003), Gourma and Jia (2015), for example. In a three dimensionnal linear pipe, a CFD approach with a level set model is adopted in Lakehal et al (2012) while a Volume of Fluid (VOF) model described in Bartosiewicz et al (2008), Febres and Nieckele (2010) and more recently in $\mathrm{Lu}$ (2015), is validated for predicting a slug flow regime.

Engineers have designed new forms of tubing that can be used as separators in multiphase flows or as slug regime controllers. Such sub-systems can be used as a compact conditioning device (Di Matteo, 2003; Vidnes and Engvik, 2014). The flow regime can be altered by damping slugs partly or totally. In industrial applications, pipelines usually have diameters greater 
than $50 \mathrm{~mm}$ with elongated coiled sub-systems and low amplitude ratio with high curvatures.

This work is not directed to perform statistical analysis on slug characteristics such as frequencies, lengths and velocities. The objective is to gain some insight into slug flow interactions with helical sub-systems through the investigation of several aspects: i.) analyse the slug frequency alterations based on the length of the helical devices, ii.) establish qualitative correlations between mean tangential velocity and helicity density fluctuations, iii.) justify the appearance of small time scales (high frequencies) and large time scales (low frequencies) in the mean helicity density histograms and their link to flow regimes, iv.) analyse the gas-liquid helicity density to predict zones of low helicity intensity (high turbulence intensity).

Simulations described here were conducted with the volume of Fluid model (VOF) and the $k-\epsilon$ turbulence model embedded in the CFD package Fluent (2006).

\section{Mathematical Model}

\subsection{The Volume of Fluid Model}

The volume of fluid model originated by Nichols et al. (1984) is implemented in most commercial CFD software. This model is based on the assumption that two or more fluids are not inter-penetrating. Variables and properties in each cell are functions of the phase fractions, as detailed in the Fluent (2006) user guide. Based on this definition, the continuity equations for liquid and gas volume fractions $\alpha_{l}$ and $\alpha_{g}$ can be written as:

$$
\partial_{t} \alpha_{i}+\nabla \cdot\left(\alpha_{i} \vec{u}\right)=0
$$


where the subscript $i$ denotes either the liquid $(l)$ or the gas $(g)$ phase. The momentum equation uses a single velocity field $\vec{u}$ acting on the mixture with a density $\rho=\alpha_{g} \cdot \rho_{g}+\alpha_{l} \cdot \rho_{l}$ and a viscosity $\mu=\alpha_{G} \cdot \mu_{g}+\alpha_{l} \cdot \mu_{l}$.

The momentum balance in conservative form is:

$$
\partial_{t} \rho \vec{u}+\nabla \cdot(\rho \vec{u} \vec{u})=-\nabla p+\nabla \cdot \tau+\rho \cdot \vec{g}+\rho \cdot \vec{F}
$$

where, $\tau$ is the deviatoric stress tensor, given by:

$$
\tau=\mu \cdot\left(\nabla \vec{u}+\nabla \vec{u}^{T}\right)
$$

with $p$ the pressure. The last term on the right hand side of Equation 2 represents the interfacial surface tension $F$ between phases which can be expressed as:

$$
F=\sigma_{i j} \cdot \frac{\kappa_{i} \cdot \nabla \alpha_{i}}{\frac{1}{2}\left(\rho_{i}+\rho_{j}\right)}
$$

and Indices $i$ and $j$ represent again the two phases, $\sigma_{i j}$ is the surface tension coefficient, and $\kappa_{i}$ is the curvature at the interface where the surface tension is calculated.

\subsection{The $k-\epsilon$ Turbulence Model}

The $k-\epsilon$ turbulence scheme belongs to the two-equation eddy-viscosity turbulence model family. It has been used in industry for decades due to its good compromise between numerical demands and stability. The scheme is semi-empirical. All fields are decomposed as $\vec{\psi}=\bar{\psi}+\vec{\psi}^{\prime}$, where the first term stands for the large scale (averaged) and the second term represents the fluctuating part. Hence two additional transport equations must be solved 
to compute the Reynolds stresses: the first one for the turbulent kinetic energy $k$, and the second for the rate of turbulence dissipation $\epsilon$ :

$$
\begin{gathered}
\partial_{t} \rho \cdot k+\nabla \cdot(\rho \bar{u} k)=\nabla \cdot\left(\left(\mu+\frac{\mu_{t}}{\sigma_{k}}\right) \nabla k\right)+G_{k}-\rho \cdot \epsilon \\
\partial_{t} \rho \cdot \epsilon+\nabla \cdot(\rho \bar{u} \epsilon)=\nabla \cdot\left(\left(\mu+\frac{\mu_{t}}{\sigma_{\epsilon}}\right) \nabla \epsilon\right)+C_{1} \cdot \frac{\epsilon}{k} \cdot G_{k}+C_{2} \cdot \rho \cdot \frac{\epsilon^{2}}{k}
\end{gathered}
$$

Quantities $C_{1}, C_{2}, \sigma_{\epsilon}$ and $\sigma_{k}$ are empirical constants. The turbulent viscosity $\mu_{t}$ is derived from $k$ and $\epsilon$ and involves an experimental constant $C_{\mu} \simeq 0.09$

$$
\mu_{t}=\rho \cdot C_{\mu} \cdot \frac{k^{2}}{\epsilon}
$$

The source term of turbulence $G_{k}$ appearing in Equations 5 and 6 , is a function of the turbulent viscosity and velocity gradients:

$$
G_{k}=\mu_{t} \cdot\left(\nabla \bar{u}+\nabla \bar{u}^{T}\right) \cdot \nabla \bar{u}^{T}-\frac{2}{3} k \cdot \nabla \bar{u}
$$

Transport equations are solved for $k$ and $\epsilon$, the turbulent viscosity $\mu_{t}$ is computed and the Reynolds stresses are determined and substituted into the momentum equations. The new velocity components are used to update the turbulence generation term $G_{k}$, and the process is repeated.

\section{Helicity fluctuations and turbulence}

The importance of helicity in turbulence was first recognised in plasma physics. The helicity density $h(r, t)$ is a pseudo-scalar defined locally (Moffatt, 1969) as $h=\vec{u} \cdot \vec{\omega}(r, t)$. It is interpreted as a measure of the entanglement of the vorticity field lines $\vec{\omega}(r, t)=\nabla \wedge \vec{u}(r, t)$.

The total helicity over the material volume (control) bounded with the surface $\partial D$ on which $\left.\vec{\omega} \cdot \vec{n}\right|_{\partial D}=0$ is given by:

$$
\mathcal{H}_{D}(t)=\int_{D} \vec{u}(r, t) \cdot \vec{\omega}(r, t) \cdot d^{3} r
$$


To draw attention on the role of helicity in turbulence (energy cascade process), the momentum balance in non conservative form is considered. Taking into account the mass conservation for the mixture density $\rho(r, t)$ and rearranging the non-linear term into $\left.(\vec{u} \cdot \nabla) \vec{u}=\vec{\omega} \wedge \vec{u}+\frac{1}{2} \nabla \cdot \vec{u} \vec{u}\right)$ in the acceleration balance, Equation 2 is reformulated as:

$$
\rho \cdot\left(\partial_{t} \vec{u}+\vec{\omega} \wedge \vec{u}+\frac{1}{2} \nabla \cdot \vec{u} \vec{u}\right)=-\nabla \cdot \sigma+\rho \cdot \vec{g}+\rho \cdot \vec{F}
$$

Using the identity matrix $I_{d}$, the stress tensor is $\sigma=p I_{d}-\tau$. With some manipulations the momentum balance above can be re-written as:

$$
\partial_{t} \vec{u}+\vec{\omega} \wedge \vec{u}=-\nabla \cdot\left(\frac{\sigma}{\rho}+\frac{1}{2} \vec{u} \vec{u}\right)+\left(\frac{\sigma}{\rho^{2}}\right) \nabla \rho+\vec{g}+\vec{F}
$$

The second term on the right hand side of Equation 11 vanishes everywhere except in the vicinity of the gas-liquid interface. Assuming an incompressible interface with a constant density, $\bar{\rho}=\left(\rho_{l}+\rho_{g}\right) / 2$, it follows $\nabla \rho=0$.

An energy cascade to small scales is an inherent aspect of turbulence in classical mechanics (Chen et al., 2003). It was stated by Hussain (1986), and Rogers and Moin (1987) that the inception of turbulence results from the non-linear term $(\vec{\omega} \wedge \vec{u})$ becoming dominant over the linear dissipative term $(\nabla \cdot \tau)$. The non-linear term is related to the helicity via the following trigonometric relation:

$$
(\vec{u} \cdot \vec{\omega})^{2}+(\vec{\omega} \wedge \vec{u})^{2}=|u|^{2} \cdot|\omega|^{2}
$$

This relation indicates that the high helicity density $(\vec{u} \cdot \vec{\omega}= \pm|u||\omega|)$ tends to inhibit the non-linear interactions (convection) and therefore minimises the magnitude of the energy cascade to small scales.

In the present work, the mean helicity density per unit surface over a surface $S_{b}(x)$ of axial coordinate $x$ is monitored. Following Reynolds' 
decomposition, the helicity density can be approximated into a mean and a fluctuating part:

$$
\begin{aligned}
\mathcal{H}(x, t) & =\left\langle\bar{u} \cdot \bar{\omega}(r, t)+u^{\prime} \cdot \omega^{\prime}(r, t)\right\rangle_{S_{b}} \\
& =\left(\int_{S_{b}} \bar{u} \cdot \bar{\omega}(r, t) \cdot d S+\int_{S_{b}} u^{\prime} \cdot \omega^{\prime}(r, t) \cdot d S\right) / S_{b}(x) \\
& =\overline{\mathcal{H}}(x, t)+\mathcal{H}^{\prime}(x, t)
\end{aligned}
$$

The helicity density is expressed with $h=u_{i} \cdot \omega_{i}=u_{i} \cdot \epsilon_{i j k} \partial_{k} u_{j}$. Its fluctuating part is represented by $h^{\prime}=u_{i}^{\prime} \cdot \omega_{i}^{\prime}=u_{i}^{\prime} \cdot \epsilon_{i j k} \partial_{k} u_{j}^{\prime}$ which is related to the Reynolds stress $R_{i j}=u_{i}^{\prime} \cdot u_{j}^{\prime}$, where $\epsilon_{i j k}$ is the Levi-Civita unit tensor. The instantaneous helicity $\mathcal{H}(x, t)$ fluctuates and can take positive and negative values.

\section{Numerical Simulations}

Petrochemical industries use coiled sub-systems of curvatures five to ten times larger with an amplitude ten times smaller than those usually studied and reported in the literature. The different geometries used in this work are described in the following section; their size correspond to devices present in industrial applications.

\subsection{Helical devices geometries}

Helical coiled devices are manufactured by meandering a pipe of diameter $d$ around a cylinder ofSimulation and experimental study of phase segregation in helical pipes diameter $|D-d|$ at a constant elevation $\lambda$ per revolution along the cylinder, as schematised in Figure 1.

The dimensional parameters characterising an helix are the curvature $\delta$ and the torsion $\tau$ which are function of the pitch, $\lambda$, which is the height of one complete helix turn, measured parallel to the axis of the helix:

$$
\delta=d /\left(D^{2}+\lambda^{2}\right) \quad \text { and } \quad \tau=\lambda /\left(D^{2}+\lambda^{2}\right)
$$


Long pipes $(L \approx 40 D)$ are needed for slug flow simulations. Predictions with short pipes can lead to incorrect slug frequencies (Lakehal, 2011) and therefore to erroneous slug flow characterisations. In addition, since slug flows are asymmetric and highly intermittent, 3D geometries are required and only statistically averaged parameters such as slug frequencies, lengths and translational velocities are meaningful.

Two-phase air water simulations were performed in a number of helical geometries comprising body lengths of $\mathrm{L}=3 \mathrm{~m}, 6 \mathrm{~m}$ and $12 \mathrm{~m}$ with an amplitude ratio $\alpha_{R}=1.0$. The sketches of several coiled lines are shown in Figures 1 and 2. Figure 1 shows a helical system assembled in three parts: a $1 \mathrm{~m}$ long inlet part, a $3 \mathrm{~m}$ long helix body and a $3 \mathrm{~m}$ long outlet section. Figure 2 shows the unstructured mesh generated for such systems for helix body lengths of $6 \mathrm{~m}$ and $12 \mathrm{~m}$. All systems investigated in this work have the

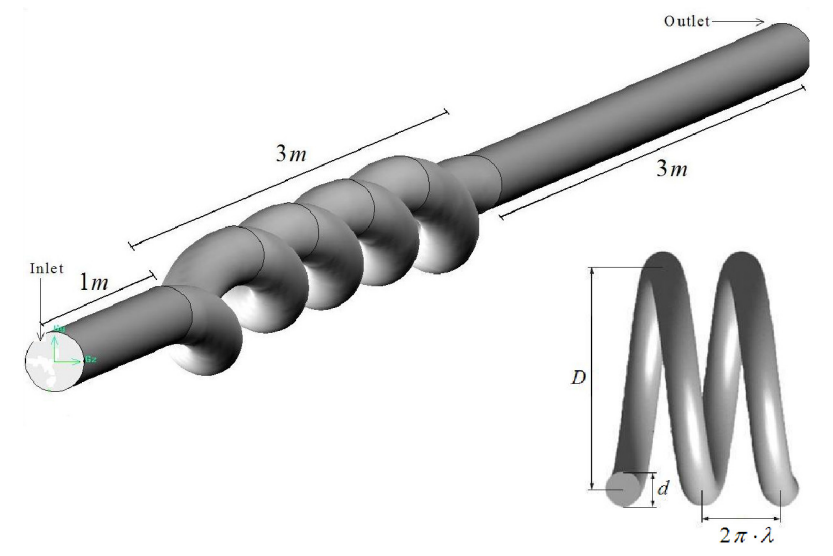

Figure 1: 3D Helical system of $\mathrm{L}=3 \mathrm{~m}$ helix body length.

same inlet and outlet lengths: $1 \mathrm{~m}$ and $3 \mathrm{~m}$, respectively. Only the helix body lengths were modified accordingly. Note that in the following the sub-system denotes either the $3 \mathrm{~m}, 6 \mathrm{~m}$ or the $12 \mathrm{~m}$ long helix while the system denotes the $1 \mathrm{~m}$ long straight pipe upstream of the sub-system, the sub-system itself 


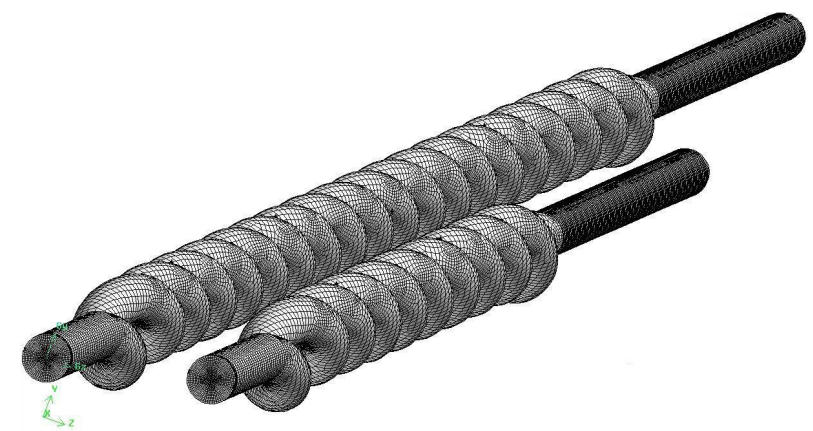

Figure 2: $6 \mathrm{~m}$ long (right) and 12m long (left) helices.

and the $3 \mathrm{~m}$ long straight pipe downstream of the sub-system.

\subsection{Methodology}

Slug flow simulations have initially been carried out with the VOF model in relatively long linear pipe of length $L=30 \mathrm{~m}$ and of diameter $D=$ $0.078 \mathrm{~m}$. These simulations in straight pipes were totally separated from the current ones performed in helical systems. The straight pipe diameter was however identical to the inlet diameter of the helical systems. Flow field data (pressure, velocity, volume fraction, turbulent kinetic energy and turbulent intensity of each phase) have been recorded during the process at $x=27 \mathrm{~m}$ every $10^{-3} \mathrm{~s}$.

Due to the nature of slug flows, the choice of the time-step is critical to capture the flow behaviour, especially in the vicinity of the liquid-gas interface. Multiphase flow simulations in 3D geometries are however very expensive in terms of CPU time and memory. Even if such a time-step might seem too large to capture all features of the flow, several initial tests have been performed before running the full simulation. A compromise had to be established to speed-up the calculation to an acceptable level without missing crucial information. The same constant time-step $\Delta t_{\text {lin }}=10^{-3}$ s was 


\begin{tabular}{ccc} 
Case reference & \multicolumn{2}{c}{ Superficial velocity } \\
& Gas & Water \\
& $U_{S G}[\mathrm{~m} / \mathrm{s}]$ & $U_{S L}[\mathrm{~m} / \mathrm{s}]$ \\
\hline Case 1 & 4.016 & 0.519 \\
Case 2 & 8.03 & 1.04 \\
Case 3 & 8.0 & 0.6
\end{tabular}

Table 1: Initial flow conditions

finally applied to the helical systems when injecting the data extracted from the separate straight pipe simulation for a period of time typically between 30 and 40 seconds (real time).

\section{Simulation Results}

The flow conditions used in this work are reported in Table 1. An isothermal two-phase air and water system at atmospheric pressure with a surface tension $\sigma_{i j}=0.072 \mathrm{~N} / \mathrm{m}$ is considered. According to the flow map from Manolis (1995), all cases investigated here are in the slug flow regime. Experiments relative to Case 1 were performed by Manolis (1995) while experiments relative to Cases 2 and 3 were conducted by Fisher et al. (2009). Simulations were performed under these conditions and the water volume fraction $\alpha_{l}$ was plotted at different positions down the system, including the inlet section. To prevent potential outlet effects commonly encountered in CFD simulations, $\alpha_{l}$ was monitored $0.5 \mathrm{~m}$ before the outlet section of the whole system. Tangential mixture velocities and helicity densities were recorded immediately after the helix body, this is discussed in details in Section 5.2. 


\subsection{Slug frequency alteration}

The redistribution of gas and liquid through different geometries is analysed in terms of slug frequency alterations induced by the sub-systems under specific flow conditions. As a reminder the sub-system denotes either the $3 \mathrm{~m}, 6 \mathrm{~m}$ or the $12 \mathrm{~m}$ long helix.

The slug frequency is a characteristic of a regime and is estimated as the ratio of the number of slugs crossing a pipe section per second. Slug frequencies at the outlet section of the helix (more precisely $0.5 \mathrm{~m}$ before the outlet) are determined and compared to those obtained at the inlet section of the sub-systems for all three cases listed in Table 1.

\subsubsection{Test case $1, U_{S G}=4.016 \mathrm{~m} / \mathrm{s}, U_{S L}=0.519 \mathrm{~m} / \mathrm{s}$}

The first case investigated (Case 1) has the lowest flow velocities among all cases investigated in this work. Figure 3 shows the inlet and outlet traces of water volume fraction for helices lengths of $3 \mathrm{~m}, 6 \mathrm{~m}$ and $12 \mathrm{~m}$.

To isolate travelling waves from stable slugs, a liquid peak is considered and accounted for a slug when the liquid volume fraction is found higher or equal to $\alpha_{l}=0.8$. Based on this approach, the slug frequency at the inlet section is $\mathcal{N}_{\text {in }} \simeq 0.34 \mathrm{~s}^{-1}$. It can be seen that such a helical system can reduce the number of slugs and its efficiency increases with the reduction of the helix length. Outlet frequencies are estimated around $\mathcal{N}_{\text {out }}=0.21 \mathrm{~s}^{-1}$, $\mathcal{N}_{\text {out }}=0.24 \mathrm{~s}^{-1}$ and $\mathcal{N}_{\text {out }}=0.26 \mathrm{~s}^{-1}$ for the $3 \mathrm{~m}, 6 \mathrm{~m}$ and $12 \mathrm{~m}$ helices, respectively. This shows a reduction of slugs around $38 \%, 29 \%$ and $24 \%$ for the $3 \mathrm{~m}, 6 \mathrm{~m}$ and $12 \mathrm{~m}$ long helices, respectively.

A helical system could also be used as a flow separator. Figure 4 shows the $3 \mathrm{D}$ contour plot of the volume fraction of air for the $12 \mathrm{~m}$ long helix, where the red color represents pure air, the blue color represents pure water 


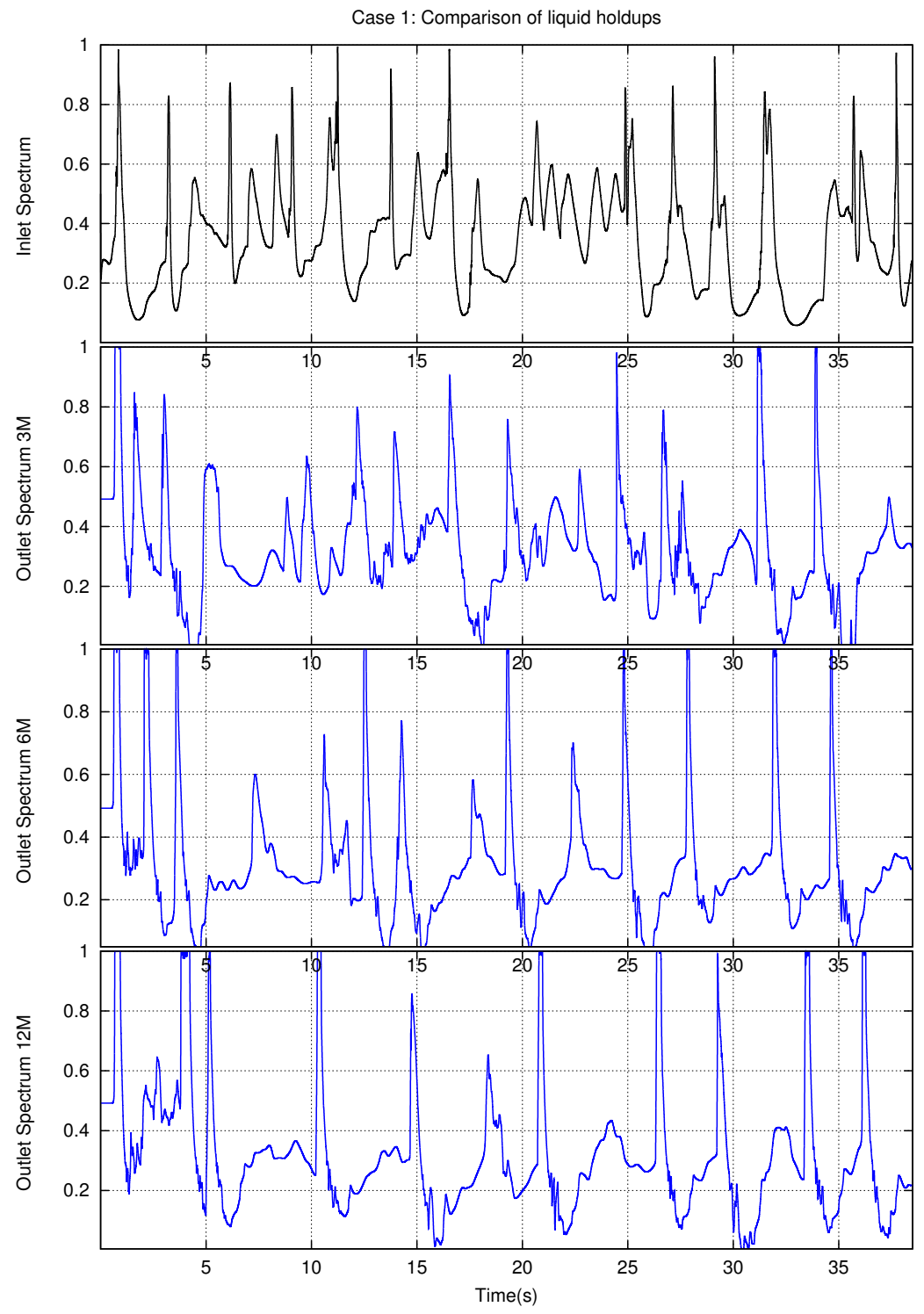

Figure 3: Inlet and outlet liquid volume fraction for $3 \mathrm{~m}, 6 \mathrm{~m}$ and $12 \mathrm{~m}$ long helices. Case 1: $U_{S G}=4.016 \mathrm{~m} / \mathrm{s}, U_{S L}=0.519 \mathrm{~m} / \mathrm{s}$

and the turquoise color the liquid-gas interface. In this figure, a slug body is apparent, followed by a large core gas region and an annular liquid pattern.

Simulation results for this case show clearly that the transition to a slug- 


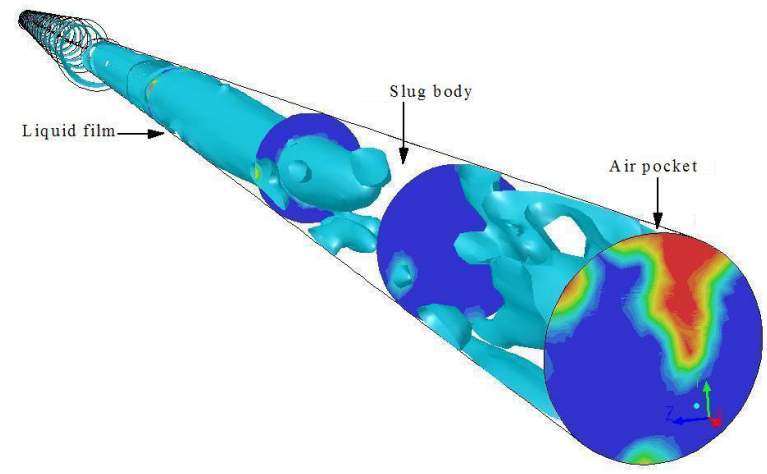

Figure 4: Snapshot of air and water distributions downstream of the $12 \mathrm{~m}$ long helix $(\mathrm{t}=27.0 \mathrm{~s})$. Red color: pure air, blue color: pure water, turquoise color: air-water interface

plug flow regime is obtained. 


\subsubsection{Test case 2, $U_{S G}=8.03 \mathrm{~m} / \mathrm{s}, U_{S L}=1.04 \mathrm{~m} / \mathrm{s}$}

The second case investigated is characterised by higher velocities than for Case 1. The slug frequency at the inlet section $\mathcal{N}_{\text {in }}=0.53 \mathrm{~s}^{-1}$ is therefore also higher than in the previous case. When comparing the water volume fraction, it appears that the values at the outlet show larger variations than at the inlet, see Figure 5. The inlet volume fraction is rarely below 0.2 while outlet values below 0.2 appear frequently for all three helix sub-systems. The flow exhibits a transition to faster fluctuations after $12 \mathrm{~s}$ in the $3 \mathrm{~m}$ long helix. A similar transition is visible after $25 \mathrm{~s}$ in the $12 \mathrm{~m}$ long device. However, no such increase of fluctuations takes place during the simulation performed with the $6 \mathrm{~m}$ long helix.

Once again, the shortest device is more efficient in reducing the number of slugs: $\mathcal{N}_{\text {out }}=0.25 \mathrm{~s}^{-1}, \mathcal{N}_{\text {out }}=0.47 \mathrm{~s}^{-1}$ and $\mathcal{N}_{\text {out }}=0.39 \mathrm{~s}^{-1}$ for the $3 \mathrm{~m}$, $6 \mathrm{~m}$ and $12 \mathrm{~m}$-long helices. This corresponds to a reduction of the number of slugs around $53 \%, 11 \%$ and $26 \%$ for the $3 \mathrm{~m}, 6 \mathrm{~m}$ and $12 \mathrm{~m}$ helix sub-systems, respectively. Surprisingly, the largest helix seems more efficient in reducing the number of slugs than the medium one. At that stage, it is difficult to explain this phenomenon, this could be case specific. 


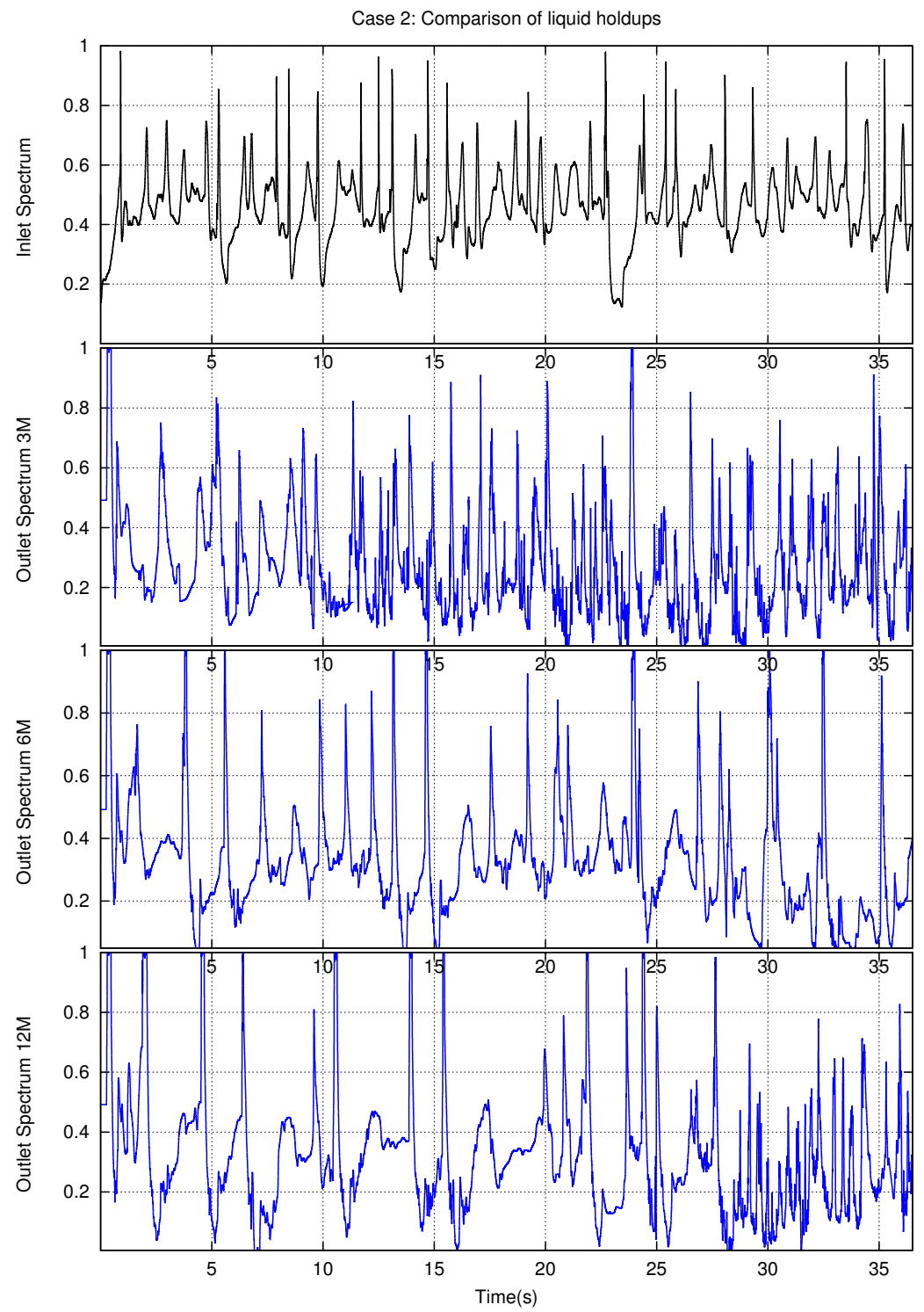

Figure 5: Inlet and outlet liquid volume fraction for $3 \mathrm{~m}, 6 \mathrm{~m}$ and $12 \mathrm{~m}$ long helices. Case 2: $U_{S G}=8.03 \mathrm{~m} / \mathrm{s}, U_{S L}=1.04 \mathrm{~m} / \mathrm{s}$

\subsubsection{Test case 3, $U_{S G}=8.0 \mathrm{~m} / \mathrm{s}, U_{S L}=0.6 \mathrm{~m} / \mathrm{s}$}

For the third case, the inlet slug frequency is $\mathcal{N}_{i n} \simeq 0.2 \mathrm{~s}^{-1}$. The volume fraction at the outlet plotted for the $3 m$ long helix in Figure 6 exhibits fast 
variations. This intermittent behaviour does not characterise a slug flow

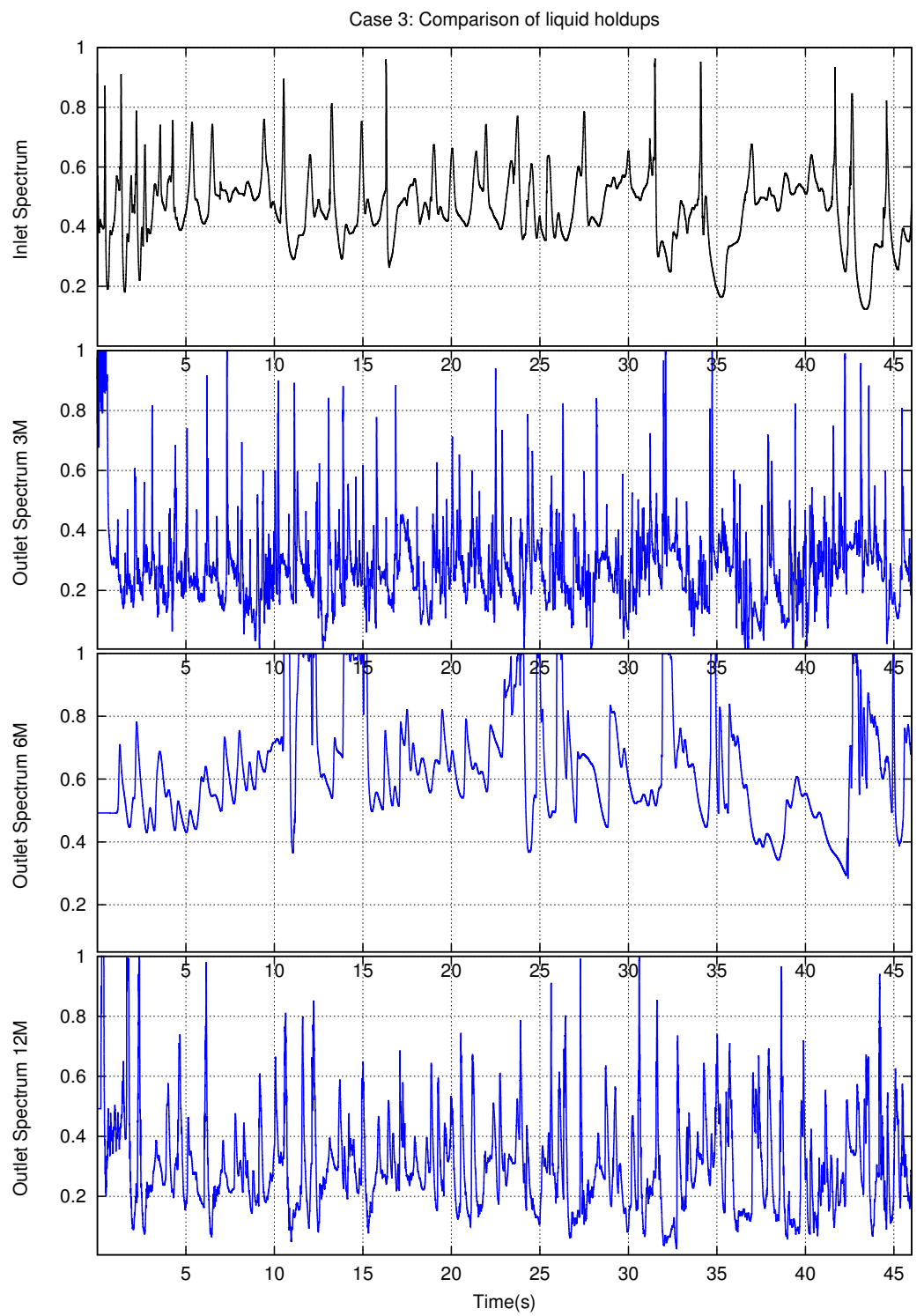

Figure 6: Inlet and outlet liquid volume fraction for $3 \mathrm{~m}, 6 \mathrm{~m}$ and $12 \mathrm{~m}$ long helices. Case 3: $U_{S G}=8.0 \mathrm{~m} / \mathrm{s}, U_{S L}=0.6 \mathrm{~m} / \mathrm{s}$

regime, the peaks being too numerous and not large enough to be slugs. 3D contour plots of volume fraction are shown in Figures 7 and 8. These 


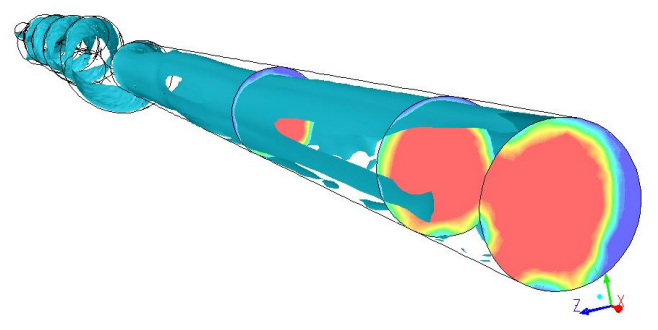

Figure 7: Snapshot of regular liquid film distribution in the $3 \mathrm{~m}$ long helix at $\mathrm{t}=28.4 \mathrm{~s}$, Case 3. Red color: pure air, blue color: pure water, turquoise color: air-water interface

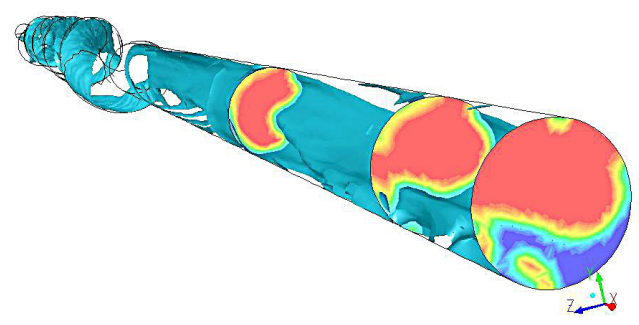

Figure 8: Snapshot liquid distribution in the $3 \mathrm{~m}$ long helix at $\mathrm{t}=32.6 \mathrm{~s}$, Case 3 . Red color: pure air, blue color: pure water, turquoise color: air-water interface

$3 \mathrm{D}$ contour plots have been selected at specific times $(\mathrm{t}=28.4 \mathrm{~s}$ and $\mathrm{t}=32.6 \mathrm{~s})$ when the volume fraction at the outlet is above $\alpha_{l}=0.8$ (see Figure 6). As can be seen, the structure of the flow indicates that it is in a critical regime. The outlet liquid peaks shown in Figure 5 reflect the continuous transitional flow regime between slug flow and annular flow with a non stable film.

For the 6m-long results displayed in Figure 6, the outlet histogram of liquid volume fraction very frequently fluctuates around 0.6, with large slug bodies or a succession of slugs. The corresponding flow structure is shown 


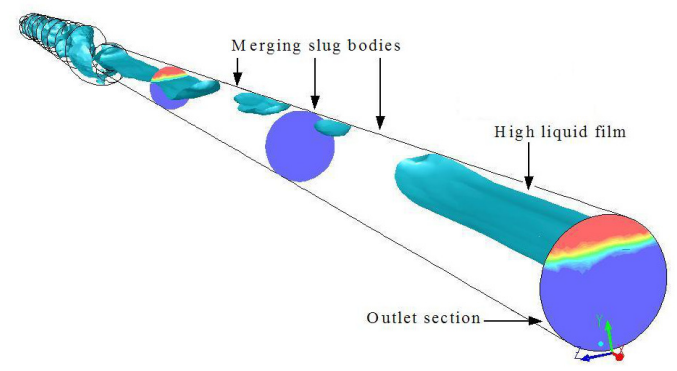

Figure 9: Snapshot of slugs travelling in the $6 \mathrm{~m}$ long helix at $\mathrm{t}=23.8 \mathrm{~s}$, Case 3. Red color: pure air, blue color: pure water, turquoise color: air-water interface

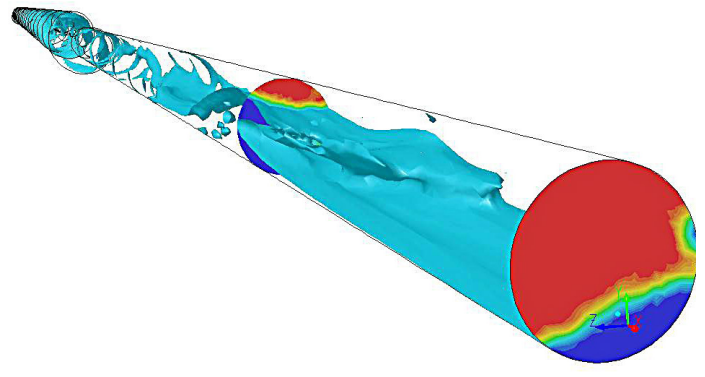

Figure 10: Snapshot of slug travelling in the $12 \mathrm{~m}$ long helix at $\mathrm{t}=31.60 \mathrm{~s}$, Case 3. Red color: pure air, blue color: pure water, turquoise color: air-water interface 
in Figure 9, where the instantaneous flow at the outlet section of the helix is visible along with two successive slugs headed by a high liquid volume fraction.

The response of the $12 \mathrm{~m}$ long helix is also plotted in Figure 6. The corresponding flow structure displayed on Figure 10 shows similarities with the one obtained with the $3 \mathrm{~m}$ long helix.

When looking at slug frequency alteration for Case 3, none of the three devices investigated behaves identically to the other one. The liquid volume fraction in the $3 \mathrm{~m}$ long helix exhibits faster fluctuations than in the $12 \mathrm{~m}$ long helix. The liquid hold-up in the $6 \mathrm{~m}$ long helix, however, shows slower variations with large slugs. Globally, the liquid height in the $6 \mathrm{~m}$ long helix is higher than in the $3 \mathrm{~m}$ and $12 \mathrm{~m}$ long helices. The outlet slug frequency in both the $3 \mathrm{~m}$ and the $12 \mathrm{~m}$ long helices is subject to high uncertainties, as it is difficult to differentiate slug bodies from sporadic fluctuations in the liquid film.

At this stage, it is unclear whether or not slug reduction is obtained under these flow conditions in either the $3 \mathrm{~m}$ or the $12 \mathrm{~m}$ long devices. The difficulty arises due to the permanent transition from the slug flow regime to the annular flow regime. Distinguishing between slugs and bursts in the liquid film renders the estimate of the slug frequency highly uncertain.

All simulation results obtained in this work are summarised in Tables 2, 3 and 4 . Table 2 compares the slug frequency in the $3 \mathrm{~m}$ long helix with available experimental data. 
Case $1 \quad$ Case $2 \quad$ Case 3

Inlet Freq $(1 / s) \quad$ Inlet Freq $(1 / s) \quad$ Inlet Freq $(1 / s)$

\begin{tabular}{cccc}
\hline Num inlet Freq & 0.34 & 0.53 & 0.2 \\
Num outlet Freq & 0.21 & 0.25 & - \\
Exp outlet Freq & 0.20 & 0.27 & 0.11 \\
\hline Num slug reduction & $38.2 \%$ & $52.8 \%$ & -
\end{tabular}

Table 2: Numerical vs Experimental frequencies: 3m long helix

$$
\text { Case } 1 \quad \text { Case } 2 \quad \text { Case } 3
$$

Inlet Freq $(1 / s) \quad$ Inlet Freq $(1 / s) \quad$ Inlet Freq $(1 / s)$

\begin{tabular}{cccc}
\hline Num inlet Freq & 0.34 & 0.53 & 0.2 \\
Num outlet Freq & 0.24 & 0.47 & 0.24 \\
\hline
\end{tabular}

\begin{tabular}{llll}
\hline Num slug reduction & $29.4 \%$ & $11.3 \%$ & -
\end{tabular}

Table 3: Numerical frequencies: $6 \mathrm{~m}$ long helix

\begin{tabular}{cccc} 
& $\begin{array}{c}\text { Case 1 } \\
\text { Inlet Freq(1/s) }\end{array}$ & $\begin{array}{c}\text { Case 2 } \\
\text { Inlet Freq }(1 / s)\end{array}$ & $\begin{array}{c}\text { Case 3 } \\
\text { Inlet Freq }(1 / s)\end{array}$ \\
\hline Num inlet Freq & 0.34 & 0.53 & 0.2 \\
Num outlet Freq & 0.26 & 0.39 & 0.26 \\
\hline Num slug reduction & $23.5 \%$ & $26.4 \%$ & -
\end{tabular}

Table 4: Numerical frequencies: $12 \mathrm{~m}$ long helix

As can be seen, slug frequencies at the outlet section of this device agree well with experimental measurements for Cases 1 and 2. However, as mentioned previously, numerical results for Case 3 are difficult to establish; they are therefore not reported in this table. Tables 3 and 4 summarise the numerical efficiency of the $6 \mathrm{~m}$ and $12 \mathrm{~m}$ long sub-systems. It appears clearly that these helix lengths are efficient in reducing the number of slugs under 
specific flow conditions. At a high superficial gas velocity rate associated with a low superficial liquid velocity, the flow becomes highly unstable and difficult to analyse. Under these specific conditions, the flow is probably in a transitional regime, from slug to annular.

\subsection{Helicity and tangential velocity fluctuations}

It is important to understand the correlations and the coupling between the fluctuating velocity and helicity density. This work aims at investigating the correlation between averaged cross sectional tangential velocity and helicity density fluctuations. For this analysis, the tangential mixture velocity and the helicity variations have been recorded across a helix section located at the outlet of the sub-systems, i.e. $3 \mathrm{~m}$ upstream of the outlet section of the whole system.

\subsubsection{Test case $1, U_{S G}=4.016 \mathrm{~m} / \mathrm{s}, U_{S L}=0.519 \mathrm{~m} / \mathrm{s}$}

Tangential velocity and helicity fluctuations are plotted on Figures 11, 12 and 13 for Case1. The signals are characterised by smaller time scale (higher frequency) than those exhibited previously in Figures 3, 5 and 6 for inlet and outlet volume fractions. The reported red ovals and red lines on all graphs indicate two categories of correlations which have been identified. In the first category, a fast positive or negative change in the tangential velocity leads to a fast positive or negative variation of the mean helicity, as indicated by the vertical red lines. 


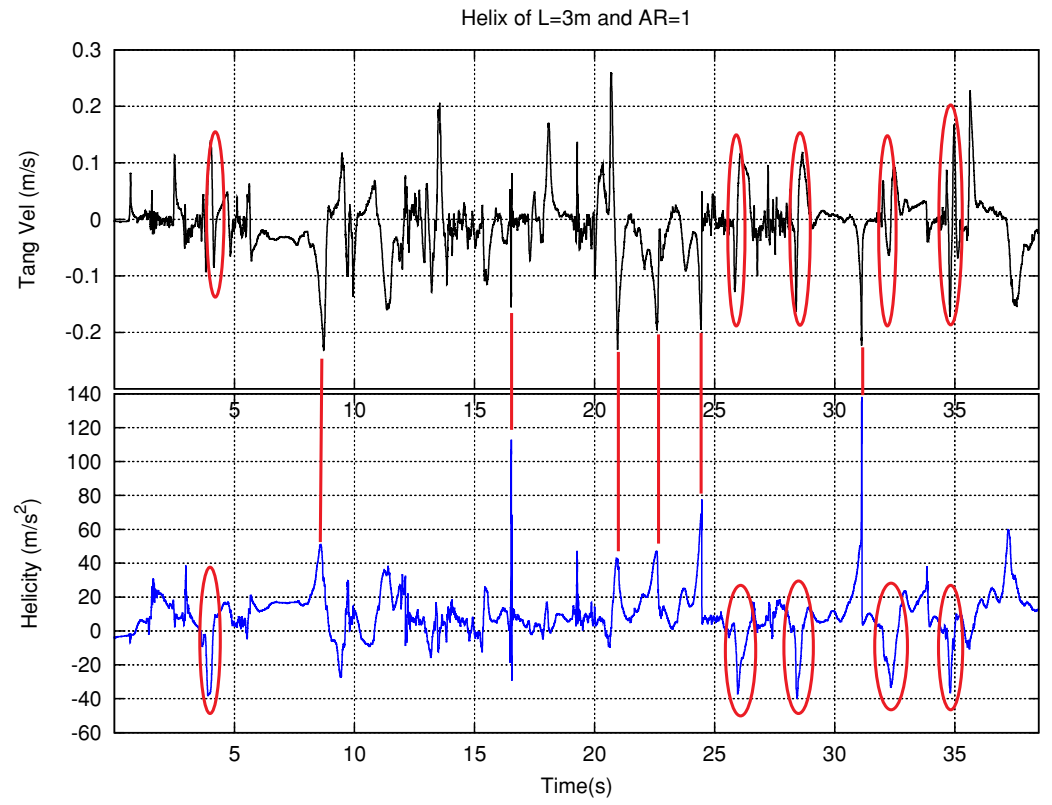

Figure 11: Histograms of the $3 \mathrm{~m}$ long helix. Case 1

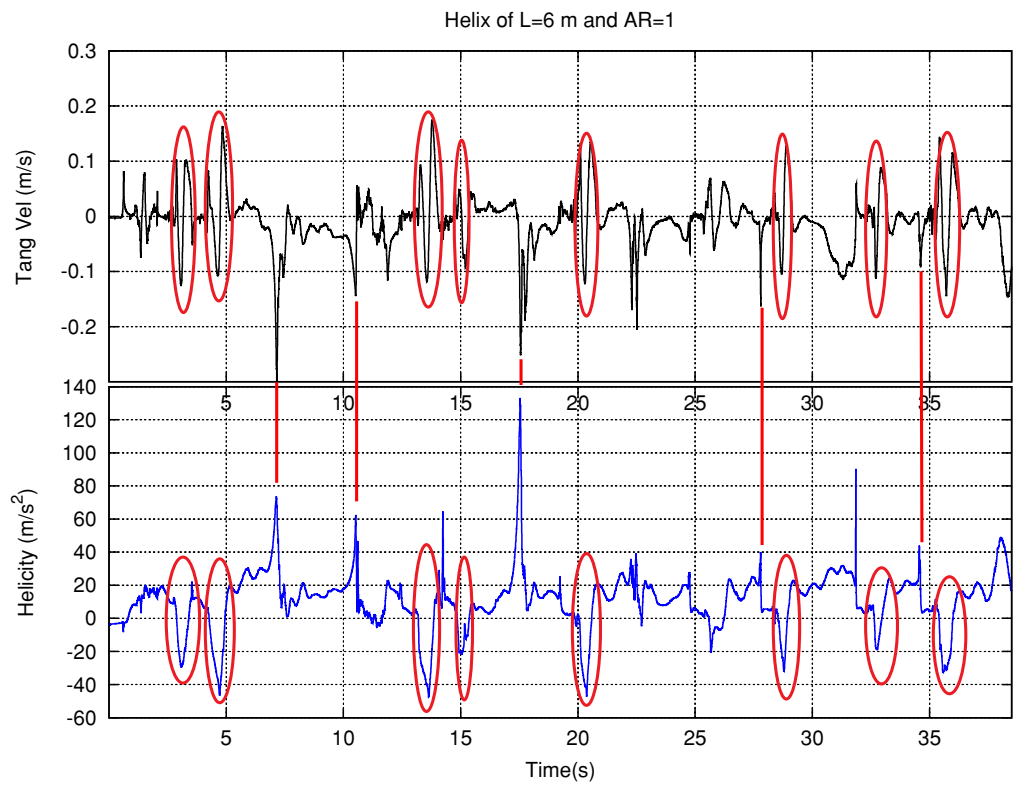

Figure 12: Histograms of the $6 \mathrm{~m}$ long helix. Case 1 


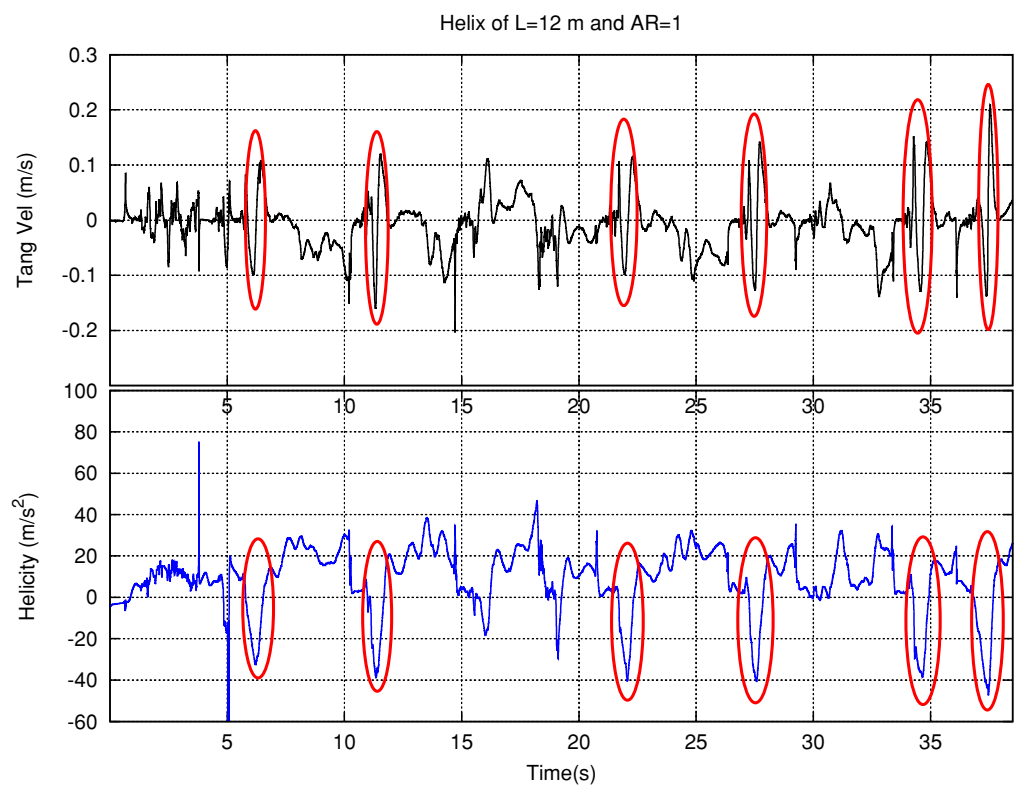

Figure 13: Histograms of the $12 \mathrm{~m}$ long helix. Case 1

For the second category, a very quick change in the orientation of the tangential velocity always produces a negative (left handed) helicity with a wide peak, as indicated with red ovals.

\subsubsection{Test case 2, $U_{S G}=8.03 \mathrm{~m} / \mathrm{s}, U_{S L}=1.04 \mathrm{~m} / \mathrm{s}$}

Tangential velocity and helicity traces are shown on Figures 14, 15 and 16 for the second test case. As in the previous case, one can notice that tangential velocity and helicity fluctuations are characterised by smaller time scale (higher frequency) than those exhibited previously in Figures 3,5 and 6 for inlet and outlet volume fractions.

Histograms in Figures 14 and 16 present a similar behaviour regarding time scale. Transitions to an even smaller time scale (higher frequency) took place at times $t=12 \mathrm{~s}$ and $t=25 \mathrm{~s}$ in the $3 \mathrm{~m}$ and $12 \mathrm{~m}$ long helices. 


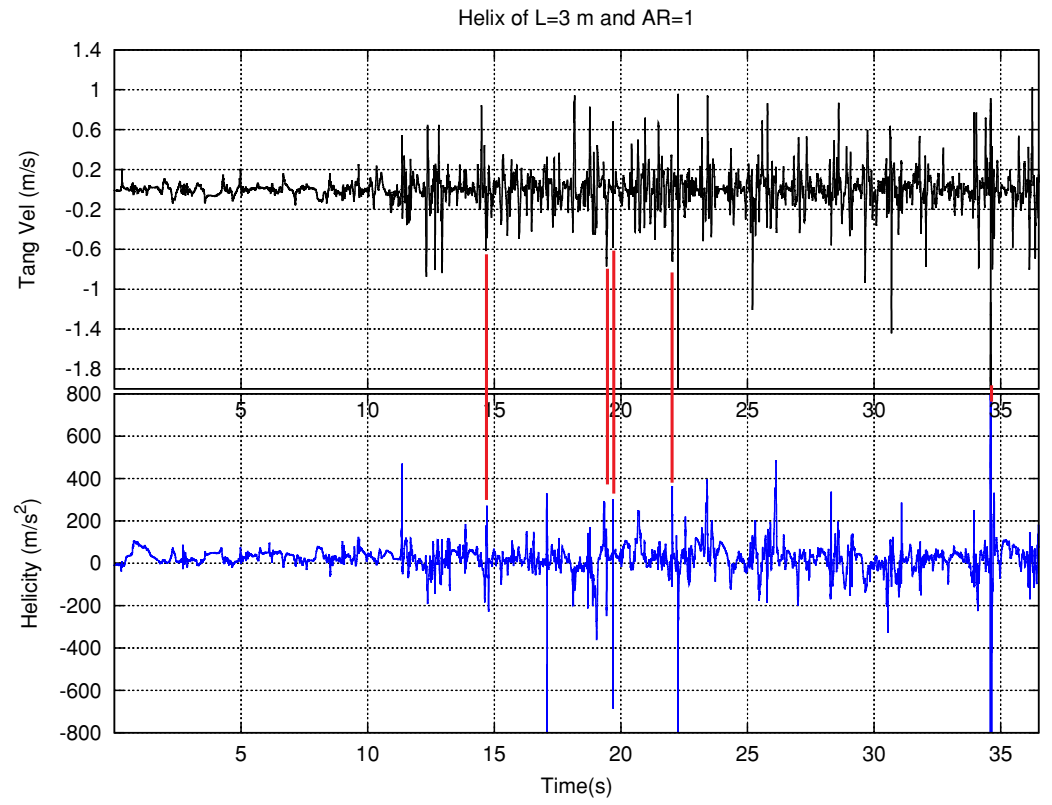

Figure 14: Histograms of the $3 \mathrm{~m}$ long helix. Case 2

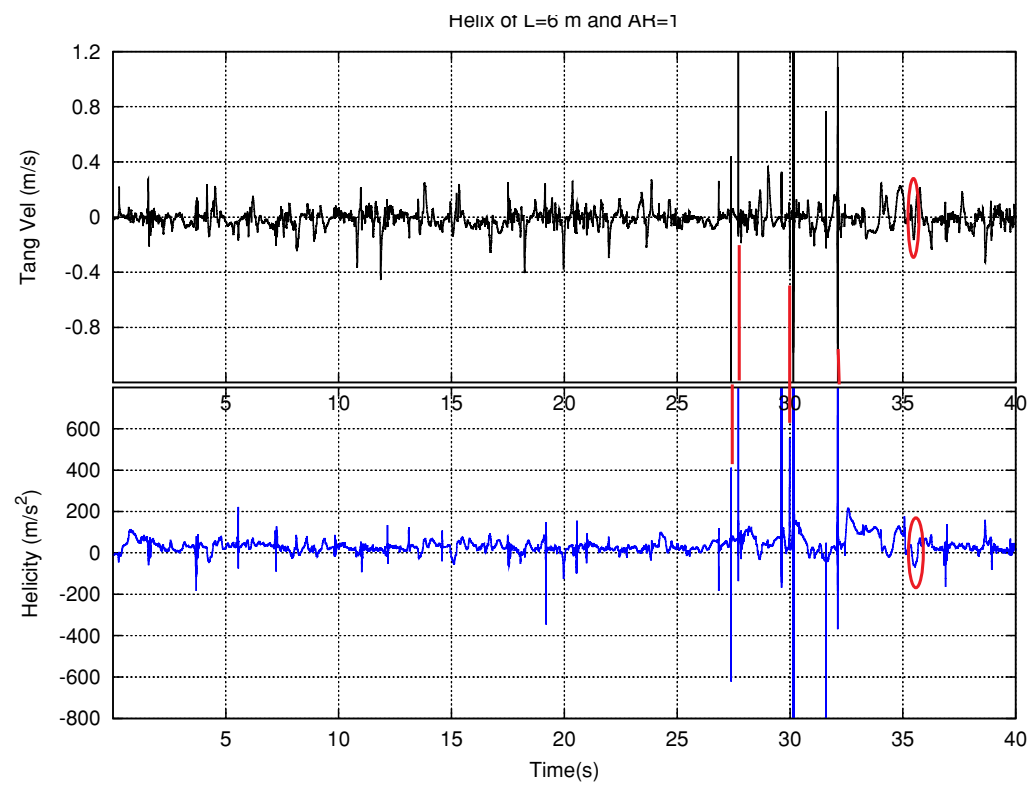

Figure 15: Histograms of the $6 \mathrm{~m}$ long helix. Case 2 


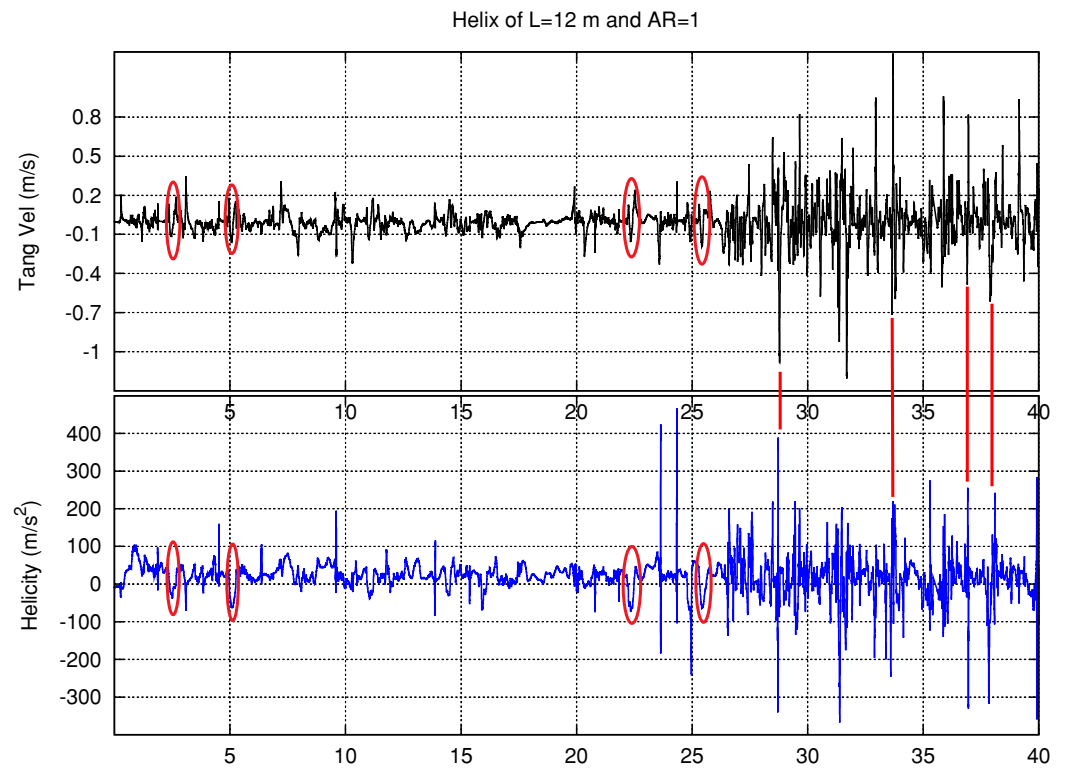

Figure 16: Histograms of the $12 \mathrm{~m}$ long helix. Case 2

Recorded correlations in these cases are mainly of first category and occur at post-transition stages (high frequency). The transition to higher frequencies advocates for a qualitative change in the slug flow structure. Such changes were not recorded in the histogram in Figure 15, where correlations in the signals are predominantly of first category.

\subsubsection{Test case 3, $U_{S G}=8.0 \mathrm{~m} / \mathrm{s}, U_{S L}=0.6 \mathrm{~m} / \mathrm{s}$}

The intermittent behaviour of the tangential velocity in Figure 17 (Case $3,3 \mathrm{~m}$ long helix) exhibits a high frequency from the start of the simulation without transitions. The reported correlations to helicity density fluctuations are again of first category. The major difference in the helicity density histogram lies in the presence of two distinct time scales in its fluctuations, see Figure 18 for a detailed view. The presence of a larger time scale (low frequency) is likely to be driven by the variations of the axial velocity rather 


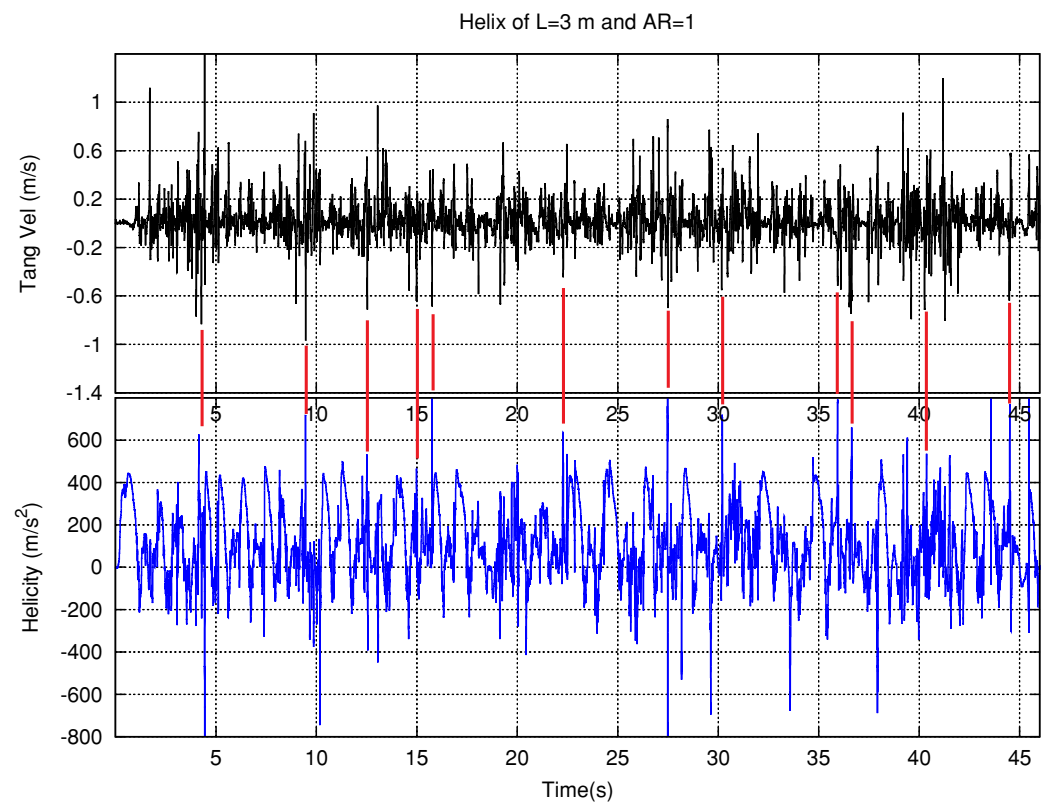

Figure 17: Histograms of 3m long helix. Case 3

than by the tangential velocity fluctuations.

The superposition of the two modes of frequency suggests that the flow is unstable and undergoes a perpetual bifurcation between two regimes. The large time scale seen in the histogram always shows a positive helicity density, suggesting that velocity and vorticty tend to be misaligned.

The examination of the fluctuating helicity densities shown in Figures 19 and 20 reveals a trend toward the alignment of vorticity and velocity fields. However, this trend is altered by sporadic jumps in the intensity of the helicity density. In addition, not all jumps are correlated to the tangential velocity intermittency. 


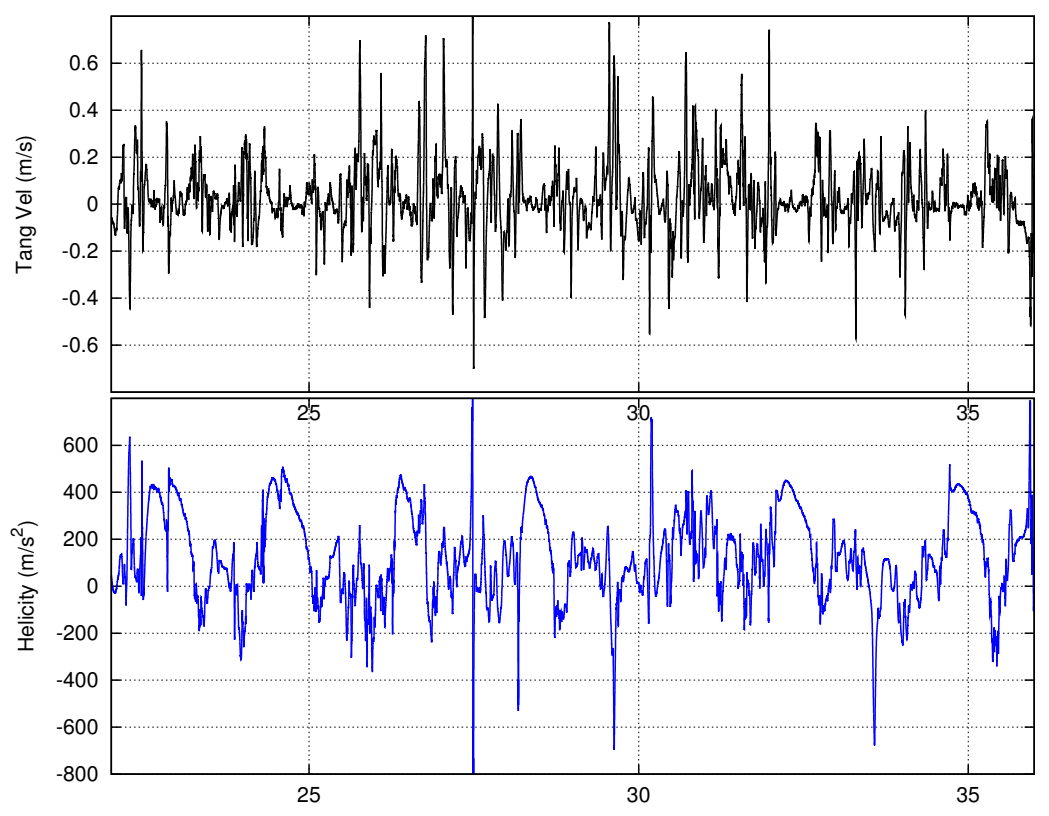

Figure 18: Closer view of the previous graph in Figure 17: 3m long helix

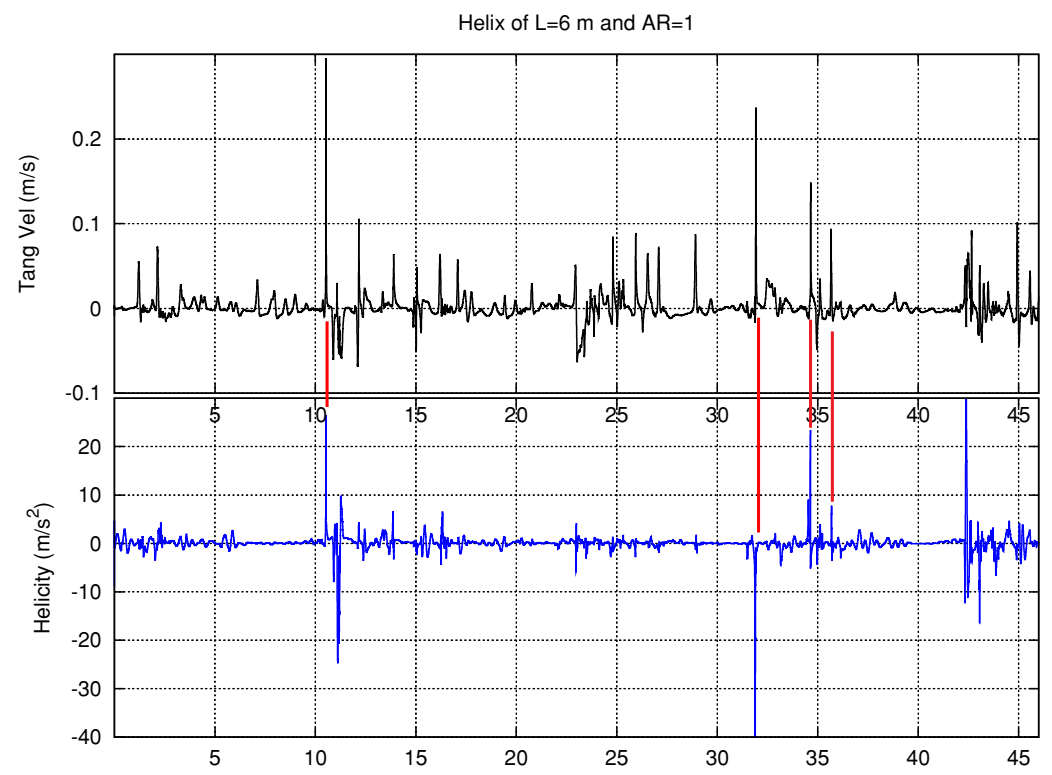

Figure 19: Histograms of the $6 \mathrm{~m}$ long helix. Case 3 


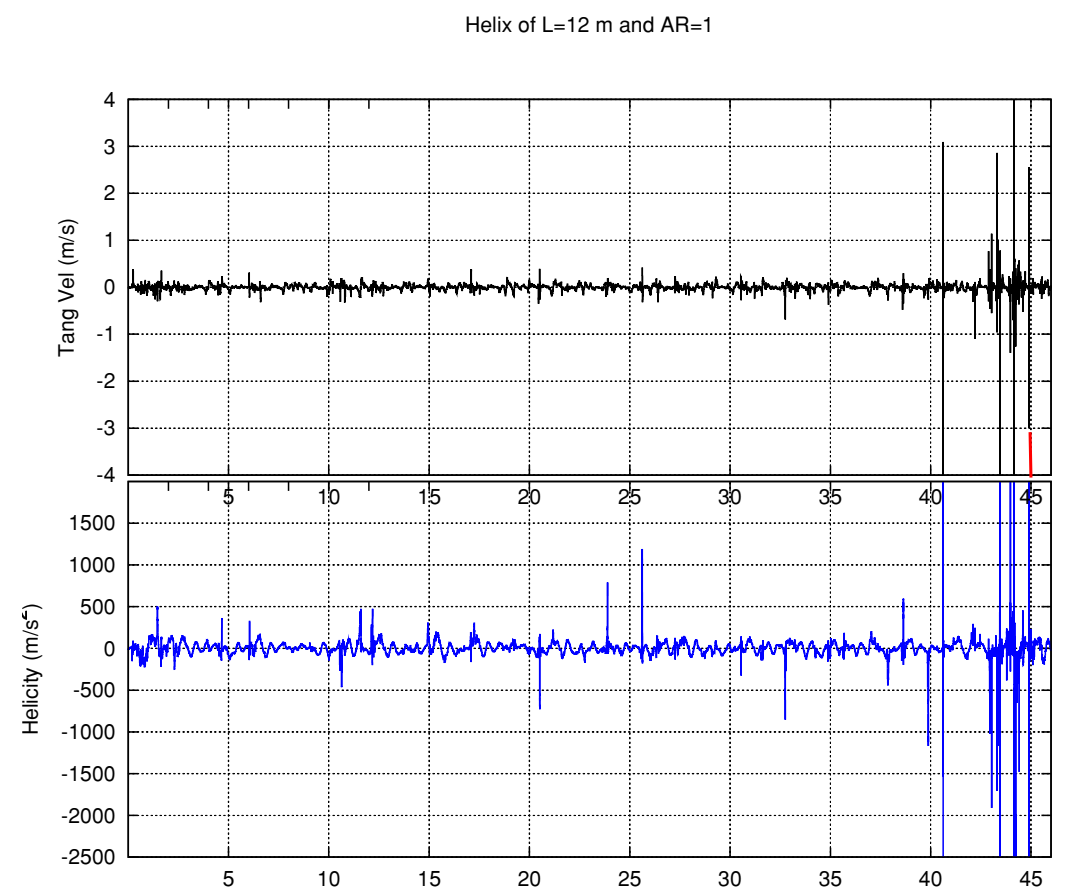

Figure 20: Histograms of the $12 \mathrm{~m}$ long helix. Case 3

To summarise, the fluctuations of tangential velocity and helicity density present smaller time scales compared to volumes fraction variations. The helicity density exhibits intermittent behaviours with persistent right handed mean helicities for Case 1, Case 2 at pre-transitions stages, and for Case 3 in the $3 \mathrm{~m}$ long helix.

The mean helicity reaches maximum peaks, they are reported in Table 5. However, the values within each case, i.e. with the same flow rates, appear very different. For Cases 1 and 2, the maximum values are reached for both the $3 \mathrm{~m}$ and the $6 \mathrm{~m}$ long helices, around $140 \mathrm{~m} / \mathrm{s}^{2}$ and $800 \mathrm{~m} / \mathrm{s}^{2}$ respectively and down to $80 \mathrm{~m} / \mathrm{s}^{2}$ and to $500 \mathrm{~m} / \mathrm{s}^{2}$ in the $12 \mathrm{~m}$ long helix for the respective cases. Case 3, however, shows large variations in the maximum mean helicity with peaks reaching values around $800 \mathrm{~m} / \mathrm{s}^{2}, 40 \mathrm{~m} / \mathrm{s}^{2}$ and $2500 \mathrm{~m} / \mathrm{s}^{2}$. 


\begin{tabular}{cccc} 
& \multicolumn{3}{c}{ Max helicity $\left[\mathrm{m} / \mathrm{s}^{2}\right]$} \\
helix length & Case 1 & Case 2 & Case 3 \\
\hline $3 \mathrm{~m}$ & 140 & 800 & 800 \\
$6 \mathrm{~m}$ & 140 & 800 & 40 \\
$12 \mathrm{~m}$ & 80 & 500 & 2500
\end{tabular}

Table 5: Maximum helicity values for all helix lengths

When looking at the tangential velocity fluctuations, the maximum peak, $4 \mathrm{~m} / \mathrm{s}$, is reached in Case 3 for the $12 \mathrm{~m}$ helix; the maximum helicity was also obtained for this case and for this helix length. Overall, the maximum peaks of tangential velocity do not exceed $0.3 \mathrm{~m} / \mathrm{s}$ for Case 1 and $1.4 \mathrm{~m} / \mathrm{s}$ for Case 2.

\subsection{Helicity analysis in VOF model}

Unstable two-phase flows in cylindrical lines produce local helicity densities. It is observed experimentally and numerically that heterogeneous flows even in linear lines are non vorticity free.

If the mixture velocity of the non-miscible fluids is $\vec{u}_{\text {mix }}=\alpha_{g} \overrightarrow{u_{g}}+\alpha_{l} \overrightarrow{u_{l}}$, then the total helicity density can be expressed as:

$$
\begin{aligned}
h(r, t) & =\vec{u}_{\text {mix }} \cdot \nabla \wedge\left(\alpha_{g} \overrightarrow{u_{g}}+\alpha_{l} \overrightarrow{u_{l}}\right) \\
& =\vec{u}_{\text {mix }} \cdot\left(\nabla \alpha_{g} \wedge{\overrightarrow{u_{g}}}+\alpha_{g} \cdot \nabla \wedge \vec{u}_{g}+\nabla \alpha_{l} \wedge \overrightarrow{u_{l}}+\alpha_{l} \cdot \nabla \wedge \vec{u}_{l}\right) \\
& =\nabla \alpha_{g} \cdot\left(\vec{u}_{g} \wedge \vec{u}_{l}\right)+\vec{u}_{m i x} \cdot\left(\alpha_{g} \cdot \nabla \wedge \vec{u}_{g}+\alpha_{l} \cdot \nabla \wedge \vec{u}_{l}\right)
\end{aligned}
$$

In the equation above, the scalar identity product, $\vec{a} \cdot \vec{b} \wedge \vec{c}=\vec{b} \cdot \vec{c} \wedge \vec{a}$ was used. At the gas-liquid interface, the volume fraction $\alpha_{k} \mapsto \alpha(r, t)$ with $k=g, l$, then the helicity becomes:

$$
h(r, t)=\nabla \alpha \cdot\left(\vec{u}_{g} \wedge \vec{u}_{l}\right)+\alpha^{2}\left(\vec{u}_{g}+\vec{u}_{l}\right) \cdot \nabla \wedge\left(\vec{u}_{g}+\vec{u}_{l}\right)
$$


The first term on the right hand side of Equation 16 stands for the helicity generated by the interface. The second term contains the helicity of each phase and the helicity due to the velocities cross coupling. In the case of single phase flows, the total helicity density changes its sign across attachment or separation vortex lines. In two-phase flows, predicting the helicity density sign is complicated by the presence of the interface. Based on the statement from Hussain (1986), and Rogers and Moin (1987) in Section 3, the locations of high energy cascade are obtained where the minimum values of helicity at the gas-liquid interface are present.

The diffusion of turbulence occurring at the interface is still an open problem in mathematical modelling; the VOF model uses a single $k-\epsilon$ field for each phase. The turbulence diffusion has certainly a significant impact on the vorticity field which is repercuted on the helicity density.

For the sake of simplify, a stable slug flow is considered here. It has been widely demonstrated in Bendiksen (1984) and van Hout et al. (2002), that the translational slug front velocity is given by $\vec{u}_{T S}=1.29 \cdot \alpha\left(\vec{u}_{g}+\vec{u}_{l}\right)+\vec{w}_{g}$ where $w_{g}=0.54 \cdot \sqrt{g \cdot d}$. The translational slug velocity being mainly axial and assuming that it is non-rotational, $\nabla \wedge \vec{u}_{T S}=0$.

The mixture velocity can be split into $u=\left(u^{\|}, u^{\perp}\right)^{T}$, with the parallel component of the axial flow, $u^{\|}$and the perpendicular component $u^{\perp}$ contained in the cross section. The helicity density becomes:

$$
\begin{aligned}
h(r, t) & =\nabla \alpha \cdot\left(\vec{u}_{g} \wedge \vec{u}_{l}\right) \\
& =\nabla_{\|} \alpha \cdot\left(\vec{u}_{g} \wedge \vec{u}_{l}\right)^{\|}+\nabla_{\perp} \alpha \cdot\left(\vec{u}_{g} \wedge \vec{u}_{l}\right)^{\perp} \\
& =\nabla_{\|} \alpha \cdot\left(\vec{u}_{g}^{\perp} \wedge \vec{u}_{l}^{\perp}\right)+\nabla_{\perp} \alpha \cdot\left(\vec{u}_{g}^{\perp} \wedge \vec{u}_{l}^{\|}+\vec{u}_{g}^{\|} \wedge \vec{u}_{l}^{\perp}\right)
\end{aligned}
$$

where, $\nabla_{\|} \alpha$ stands for the gradient in the axial direction (flow direction, here the $\mathrm{x}$ direction) whereas $\nabla_{\perp} \alpha$ stands for the transverse gradient com- 
ponent (across the section). The transverse gradient component can also be decomposed in two components: $\nabla_{\perp} \alpha=\left(\nabla_{\perp}^{i f} \alpha, \nabla_{\|}^{i f} \alpha\right)^{T}$, where one component is parallel to the interface line and the second component is perpendicular to it. The compatibility condition at the interface requires the continuity of parallel and transverse velocities, hence, $\vec{u}_{g}^{\perp}=\vec{u}_{l}^{\perp}$ and $\vec{u}_{g}^{\|}=\vec{u}_{l}^{\|}$. Consequently $h(r, t) \mapsto 0$ at the slug front, also referred to as the mixing zone.

The flow is assumed annular with a regular liquid film in the axial direction, i.e. $\nabla_{\|} \alpha<<\nabla_{\perp} \alpha$. The transition to the annular flow regime occurs when the gas velocity becomes very hight compared to the liquid velocity. It is also assumed that $\left|\vec{u}_{k}^{\perp}\right|<<\left|\vec{u}_{k}^{\|}\right|$, with $k=g, l$. A first approximation of the helicity density would be:

$$
\begin{aligned}
h(r, t) & \approx \nabla_{\perp} \alpha \cdot\left(\vec{u}_{g}^{\perp} \wedge \vec{u}_{l}^{\|}+\vec{u}_{g}^{\|} \wedge \vec{u}_{l}^{\perp}\right)+\alpha^{2}\left(\vec{u}_{g}^{\|}+\vec{u}_{l}^{\|}\right) \cdot \nabla \wedge\left(\vec{u}_{g}^{\perp}+\vec{u}_{l}^{\perp}\right) \\
& \approx \nabla_{\perp} \alpha \cdot \vec{u}_{d}^{\perp} \wedge \vec{u}_{g}^{\|}+2 \alpha^{2} \cdot \vec{u}_{g}^{\|} \cdot \nabla \wedge\left(\vec{u}_{g}^{\perp}+\vec{u}_{l}^{\perp}\right)
\end{aligned}
$$

The continuity of the axial velocity and its non-rotational form are assumed in Equation 18, hence, $\vec{u}_{g}^{\|}=\vec{u}_{l}^{\|}$and $\nabla \wedge \vec{u}_{k}^{\|}=\overrightarrow{0}$. The transverse drift velocity $\vec{u}_{d}^{\perp}=\vec{u}_{g}^{\perp}-\vec{u}_{l}^{\perp}$ contains a circumferential and a radial component. This drift velocity accounts for the mechanisms of continuous draining and regeneration of the liquid film due to entrainment and deposition. In the absence of these mechanisms, i.e. $\vec{u}_{d}^{\perp} \mapsto \overrightarrow{0}$, the following can be written:

$$
\begin{aligned}
h(r, t) & \approx 2 \alpha^{2} \cdot \vec{u}_{g}^{\|} \cdot \nabla \wedge\left(\vec{u}_{g}^{\perp}+\vec{u}_{l}^{\perp}\right) \\
& \approx \alpha^{2} \cdot\left(\vec{u}_{g}^{\|}+\vec{u}_{l}^{\|}\right) \cdot \nabla \wedge\left(\vec{u}_{g}^{\perp}+\vec{u}_{l}^{\perp}\right)
\end{aligned}
$$

Omitting the drit velocity from the expression in 18 leads to ignoring the interface effect and to the invariance of the helicity density under the 
exchange of phase velocities. It also vanishes at the vortex lines of the liquid film and the gas phase.

\section{Conclusions}

This study has shown that among all three helices investigated, the $3 \mathrm{~m}$ long one is the most effective in reducing the number of slugs. Installing such a device upstream of large systems could therefore potentially enhance the safety of production. This conclusion is however based on a limited number of simulations.

The flow fields investigated here indicate that the mean tangential velocity fluctuations can be strongly correlated to the variations of the mean helicity density. These fluctuating fields exhibit higher frequencies in comparison to volume fraction signals regardless of the stability of the flow regime. Transitions of the intermittent mean helicity to small time scales (to high frequencies) are predicted and explained in term of flow regime transition. The helicity density histogram tends to develop slower fluctuations (lower frequency), these are essentially driven by the axial velocity variations rather than by tangential fluctuations.

The occurrence of alternating large time scale (low frequencies) with small time scale (high frequencies) fluctuations in the mean helicity density are reported, this indicates that the flow undergoes continuous regime transitions.

The analysis of the helicity density at the gas-liquid interface when using the VOF model provides valuable information on slug and annular flows. It confirms the inception of turbulence in the mixing zone (low helicity den- 
sity). It also highlights the presence of transverse drift velocities in the liquid film.

It is finally worth noting that further analyses are required with a larger range of flow rates and helix geometries (amplitude ratio and length) to validate these results and determine the efficiency of such devices for their use as slug reduction systems, and/or their use as flow separators. Such study is also required to quantify the correlations between various flow fields and to shed light on the behaviour of the helicity density during multiphase flow regime transitions.

\section{Acknowledgments}

The authors would like to thank Heliswirl.Ltd for funding and supporting this project.

\section{References}

Andreussi, P., Bendiksen, K. H. and Nydal, O. J., 1993. Void Distribution Slug Flow. Int. J. Multiphase Flow, 19: 817-828.

Bartosiewicz, Y., Laviéville, J. and Seynhaeve, J. M., 2008. A model for simulating gas bubble entrainment in two-phase horizontal slug flow. Int. J. Heat Mass Flow, 29(11):1785-1717.

Bendiksen, K. H., 1984. An experimental Investigation of The motion of Long Bubbles in Inclined Tubes. Int. J. Multiphase Flow, 10(4): 467483. 
Bonizzi, M. and Issa, R. I., 2003. A model for simulating gas bubble entrainment in two-phase horizontal slug flow. Int. J. Multiphase Flow, 29(11):1785-1717.

Brissaud, A., Frisch, U., Leorat, J., Lesieur, M. and Mazure, A., 1973. Helicity cascades in fully developed isotropic turbulence. Phys. Fluids 16(8): 1366-1367.

Chen, Q., Chen, S., Eyink, G. L. and Holm, D. D., 2014.Intermittency in the Joint Cascade of Energy and Helicity Physical Rev. Letters, The American Physical Society,90(21)214503: 1-4.

Ciofalo, M., Di Liberto, M. and Marotta, G., 2014. On the influence of curvature and torsion on turbulence in helically coiled pipes. Journal of Physics, Conference Series,501: 12-25.

Cioncolini, A., Santini, L. and Ricotti, M., 2008. Subcooled and saturated water flow boiling pressure drop in small helical coils at low pressure. Exp. Thermal Fluid ScienceJournal,32: 1301-1312.

Da Mota, F. R. M. and Pagano, D. J., 2014. Simulation and experimental study of phase segregation in helical pipes: A new method for flow conditioning Flow Meas. Instrumentation, 35: 99-108.

De Henau, V. and Raithby, G. D., 1995. Study of terrain-induced slugging in two phase flow pipelines, Int. J. Multiphase Flow, 21(3): 365-379.

Di Matteo, C. A., 2003. Mechanistic Modeling of Slug Dissipation in Helical Pipes, M.S. thesis, The University of Tulsa, Tulsa, OK. 
Di Piazza, I. and Ciofalo, M., 2011. Transition to turbulence in toroidal pipes. Journal of Fluid Mechanics, 687: 72-117.

Dukler, A. E. and Hubbard, M. G., 1975. A Model for Gas-Liquid Flow in Horizontal and Near Horizontal Tubes. Ind. Engng. Chem. Fundam., 14(4): 337-347.

Fabre, J. and Liné, A., 1992. Modeling of Two-Phase Slug Flow. Annual Review of Fluid Mechanics, 24: 21-46.

Febres, M. and Nieckele, A. O., 2010. Slug flow prediction with volume of fluid model. 13th Brazilian Congress of Thermal Sciences and Engineering, December, Uberlandia, MG, Brazil.

ANSYS Fluent, 6.3, 2006. User Guide, Fluent Inc. Lebanon, NH.

Fisher, S. A., Hale, C. P.and Hewitt, G. F., 2009. The Performance of SLUGBUSTER Tubing In Modifying Slug Flows In Horizontal Flow Systems. Chemical Engineering Department. Imperial College, London.

Germano, A., 1982. On the effect of torsion on a helical pipe flow. Journal of Fluid Mechanics, 125: 1-8.

Gopal, M. and Jepson, W. P., 1997. Development of Digital Image Analysis for The Study of Velocity and Void profiles in Slug Flow. Int. J. Multiphase Flow, 23(5): 945-965.

Gourma, M. and Jia, N., 2015. Two-fluid model for 1D Gas-liquid Slug Flows: Realizable Mean Slug Characteristics. The J. Comp. Multiphase Flows, 7(2): 57-77. 
Hurlburt, E. T. and Hanratty, T. J, 2002. Prediction of the transition from stratified to slug and plug flow for long pipes. Int. J. Multiphase Flow, 28(5): 423-431.

Hussain, A. K. M. F. 1986, Coherent structures and turbulence. Journal of Fluid Mechanics, 173: 303-356.

Hüttl, T. J. and Friedrich, R., 2000. Influence of curvature and orsion on turbulent flow in helically coiled pipes. Int. J. Heat and Fluid Flow, 21(3): 345-353.

Issa, R. I.and Kempf, M. H. W., 2003. Simulation of slug flow in horizontal and nearly horizontal pipes with the two-fluid model. Int. J. Multiphase Flow, 29(1): 69-95.

Kadri, U., Mudde R. F. and Oliemans, R. V. A., 2010. Influence of the operation pressure on slug length in near horizontal gasliquid pipe, Int. J. Multiphase Flow, 36(5): 423-431.

Kolmogorov, A. N., 1941. Local structure of turbulence in an incompressible fluid at very high Reynolds number. Doklady AN SSSR: 299-303.

Kraichnan, R. H., 1973. Helical turbulence and absolute equilibrium. Journal of Fluid Mechanics, 59: 745-752.

Lakehal, D., Labois, M. and Narayanan, C., 2012. Advances in the LargeEddy and Interface Simulation (leis) of interfacial multiphase flows in pipes, Adv. Comp. Fluid Dynamics, 12(2/3: 153-163.

Lakehal, D., 2011. Advances in the simulation of multiphase flows in 
pipelines, 8th International Conference on CFD in Oil and Gas, Metall and Proc. Ind. SINTEF Trondheim, 59, June 2011. Norway

Levich, E., Shtilman, L. and Tur, A. V., 1991. The origine of coherence in hydrodynamical turbulence, Physica A, 176: 241-296.

Levich, E., 2009. Coherence in turbulence: New perspective, Concepts of Physics, VI(3)

Lu, M., 2015. Experimental and Computational Study of Two-Phase Slug Flow. PhD thesis, Department of Chemical Engineering and Chemical Technology, Imperial College of Science, Technology \& Medicine, UK.

Mandal, S. N. and Das, S. K., 2002. Gas liquid through helical coils in horizontal orientation. The Canadian Journal of Chemical Engineering, 80:979-983.

Manolis, I. G., 1995. High pressure gas-liquid slug flow. PhD thesis, Department of Chemical Engineering and Chemical Technology, Imperial College of Science, Technology \& Medicine, UK.

Moalem Maron, D., Yacoub, N., Brauner N. and Naot,D., 1991. Hydrodynamic Mechanisms in the Horizontal Slug Pattern, Int. J. Multiphase Flow, 17(2): 227-245.

Moffatt, H. K., 1969. The degree of knottedness of tangled vortex lines. Journal of Fluid Mechanics, 35: 117-129.

Moreau, J. J., 1969. Contantes d' un flot tourbillounnaire en fluid parfait barotrope. C. R. Acad. Sci. Paris,252: 2810-2912. 
Murai, Y., Yoshikawa, S., Toda, S., Ishikawaw, M. and Yamamoto, F., J. J., 2006. Structure of air-water two-phase flow in helically coiled tubes. Nuclear Engineering and Design, 236(1):94-106

Naphon, P. and Wongwises, S., 2006. A review of flow and heat transfer characteristics in curved tubes, Renewable and Sustainable Energy Review, 10:463-490.

Narasimha, R. and Sreenivasan, K., 1979. Relaminarization of fluid flows, Advances in Applied Mechanics,19: 221-309.

Nichols, B. D., Hirt, C. W. and Hotchkiss, R. S., 1981. A Fractional Volume of Fluid Method for Free Boundary Dynamics, 7th International Conference on Numerical Methods in Fluid Dynamics. Lecture Notes In Physics, 141: 304-309.

Nydal, O. J. and Pintus S. and Andreussi, P. and Andreussi, P., 1992. Statistical character-ization of slug flow in horizontal pipes, Int. J. Multiphase Flow, 18(3): 439-453.

Paglianti, A., Goina, M. and Soldati, A., 1996. Characterization of sub regimes in two-phase slug flow, Int. J. Multiphase Flow,22(4): 783-796.

Polifke, W. and Shtilman, L., 1989. The dynamics of helical decaying turbulence, Physics of Fluid A,1(12): 2025-2033

Rogers, M.and Moin, P., 1987. Helicity fluctuations in incompressible turbulent flows, Physics of Fluids,30(9): 2662-2671. 
Saffari, H.and Moosavi, R., 2014. Numerical study of the influence of geometrical characteristics of a vertical helical coil on a bubbly flow, J. Appl. Mech. Tech. Physics, 55(6): 957-969.

Sreenivasan, K. and Strykowski, P., 1983. Stabilization effects in flow through helically coiled pipes, Experiments in Fluids, 1: 31-36.

van Hout, R., Barnea, D. and Shemer, L., 2001. Evolution of statistical parameters of gas-liquid slug along vertical pipes, Int. J. Multiphase Flow, 27(9): 1579-1602.

van Hout, R., Barnea, D. and Shemer, L., 2002. Translational velocities of elongated bubbles in continuous slug flow, Int. J. Multiphase Flow, 28(8): 1333-1350.

Vashisth, S., and Nigam, K. D. P., 2009. Prediction of flow profiles and interfacial phenomena for two-phase flow in coiled tubes, Chemical Engineering Process: Process Intensification, 48: 452-463.

Vidnes, J., and Engvik, S. A., 2014. Analysis of Compact Separation Systems and Experimental Study of Centrifugal Separation in Helically Coiled Pipes. M. S. Thesis. Department of Production and Quality Engineering. NTNU Trondheim. Norway.

Xin, R. C., Awwad, Z., Dong F. Z. and Ebadian, M. A., 1997. An experimental study of single-phase and two-phase flow pressure drop in annular helicoidal pipes, Int. J. Heat and Fluid Flow, 18(5): 482-488.

Yokoi, N. and Yoshizawa, A., 1993. Statistical analysis of the effects of helicity in inhomogeneous turbulence, Physics of Fluids A,5(2): 464-477. 
Zhao, L., Guo, L.,Bai, Y., Hou, Y. and Zhang X., 2003. Convective boiling heat transfer and two-phase flow characteristics inside a small horizontal helically coiled tubing once-through steam generator, Int. J. Heat and Mass Transfer, 46: 4779-4788. 